\title{
Cell signalling and bone remodelling Part II: Developments in the pathogenesis and principles of management of selected skeletal disease states
}

\author{
EJ Raubenheimer MChD, PhD, DSc \\ Pathology: Metabolic Bone Disease Unit, Faculty of Health Sciences, SMU, South Africa \\ C Noffke MSc \\ Faculty of Health Sciences, SMU, South Africa \\ Radiology Unit, Faculty of Health Sciences, SMU, South Africa
}

*Part I was published in SA Orthopaedic Journal Summer 2015 Vol 14 No 4

\author{
Corresponding author: \\ Prof EJ Raubenheimer \\ Pathology: Metabolic Bone Disease Unit \\ Faculty of Health Sciences \\ 0204 SMU \\ Email: ejraub@fox5.co.za \\ Tel: +27125214838 \\ Fax: +27 125215274
}

\begin{abstract}
Mapping of the bone remodelling signalling pathways contributed significantly to the establishment of a scientific basis for the development of pharmaceuticals which have the potential to induce or suppress bone formation. Enhancing bone healing and the establishment of a pre-determined skeletal phenotype are now within reach of the medical profession. This manuscript provides practitioners with an overview of recent developments in the quest for uncovering the molecular mechanisms involved in the pathogenesis of selected bone disease states and the role these discoveries play in the future management of bone healing and skeletal health.
\end{abstract}

Key words: bone remodelling, osteoporosis, metastatic bone disease, Paget's disease, osteopetrosis.

http:/ / dx.doi.org/10.17159/2309-8309/2016/v15n1a11

\section{Introduction}

The study of rare genetic skeletal diseases exposed a wealth of information on the autocrine, paracrine and endocrine control of bone metabolism. Mapping of the pathways which control bone remodelling (covered in Part $\left.I^{*}\right)$ provides exciting new possibilities in the prevention and management of common skeletal deficiency states like osteoporosis. The field of regenerative medicine is growing rapidly and it is therefore no surprise that the pharmaceutical industry is investing large sums of money in the development of patented drugs and auto-antibodies which modulate the establishment of a specific skeletal outcome. Gene delivery to bone with viral vectors, plasmids or mesenchymal stem cells is certain to develop as potent tools in the manipulation of bone and treatment of skeletal disease.
Mesenchymal bone marrow stem cells have the potential to form bone, are chemically attracted to the skeleton after peripheral administration and are therefore ideal vehicles to deliver transgenes which induce anabolic or block catabolic cytokines. ${ }^{1}$ Reconstruction of large bone segments without cortico-cancellous bone grafts, which comes with the risk of disease transmission, infection and rejection, remains the prime goal of researchers in the field of tissue engineering. Using osteoconductive matrices seeded with osteogenic progenitor cells and osteoinductive and vasoproliferative factors to achieve this goal is no longer unrealistic from a scientific point of view. ${ }^{2}$ This article is aimed at providing practitioners with insight into recent advances on the impact of modulation of cell signalling on the management of selected skeletal disease states. 


\section{Osteoporosis}

The persistent loss of bone with age ultimately culminates in osteoporosis. Although the fracture risk of young males is higher than females, in older populations significantly more women are affected than men. ${ }^{3}$ The societal consequences of osteoporosis are devastating and the cost implications of the more than 2 million fractures recorded in the USA in 2005 are projected to rise to $\$ 25$ billion by $2025 .{ }^{4}$ Commercial interest in developing patented drugs that target this disease is flourishing. The most popular medication used in the prevention of osteoporosis are drugs in the bisphosphonate group, which can be administered orally, and directly inhibit osteoclasts and indirectly decrease osteoblast activity, thereby downregulating bone metabolism. Denosumab, ${ }^{5}$ a monoclonal human antibody directed against RANKL that prevents osteoclastogenesis and bone catabolism, was approved in 2010 by the FDA (US Food and Drug Administration) for use in postmenopausal women at risk for osteoporosis. Parathyroid hormone (PTH) administration is the only FDA-approved anabolic therapy for fracture prevention in postmenopausal women. ${ }^{6}$ CD8+ T-cells produce Wnt ligand under intermittent stimulation by PTH. This activates the cananonical Wnt signalling pathway in preosteoblasts and supresses the production of sclerostin by osteocytes, facilitating osteoblast differentiation and bone formation (readers are referred to Part $\mathrm{I}^{\star}$ for more information on cell signalling involved in bone remodelling). The activation or inactivation of steps in Wnt signalling in osteoblasts can induce bone anabolism or - catabolism. Stimulation of bone formation can be achieved through auto antibodies directed against endogenous Wnt-antagonists such as Dickkopf-1 and sclerostin. Inhibition of cytoplasmic kinases involved in Wnt signalling by lithium stimulates bone formation. ${ }^{7}$ Vascular endothelial growth factor $^{8}$ (VEGF) improves vascularisation and facilitates bone formation. Suspending the osteoblast-suppressing property of serotonin with drugs which antagonise its action may be the key to the development of a novel approach in preventing osteoporotic fractures. ${ }^{9}$ Although innovative research on laboratory animals shows promising results in many of these fields of research, interference with these pathways runs the risk of long-term secondary complications such as the induction of tumours. The ultimate goal is to manipulate steps in Wnt signalling which is bone-specific, thereby negating the development of unintended secondary pathology.

\section{Inflammation}

In the past decade the accumulation of data on the influence of inflammation on the skeleton has led to the development of a dedicated field of study referred to as 'osteoimmunology'. Monocytes are attracted to a site of inflammation and induced to differentiate into macrophages which have the capacity to elaborate the osteoclastogenic nuclear factor $\kappa \mathrm{B}(\mathrm{NF}-\kappa \mathrm{B})$ ligand, RANKL. ${ }^{10}$ Although several factors released during inflammation promote osteoclast activation, RANKL and its inhibitor, osteoprotegerin (OPG), are the final downstream cytokines that control osteoclast differentiation and bone resorption (see Part $I^{\star}$ ). The SOST gene which encodes for sclerostin is the only part of the Wnt pathway expressed exclusively by osteocytes and a monoclonal antibody, which inactivates SOST, is promising as interference in other cell processes appears to be limited..$^{11}$ Inflammatory-associated bone loss not only occurs in the area of inflammation, but also through osteoclastogenic cytokines released in circulation by distant inflammations, such as rheumatoid arthritis. ${ }^{12}$ Resolution of a site of inflammatory-induced bone loss follows upon the elaboration of cytokines, such as proteins belonging to the transforming growth factor beta (TGF $\beta$ ) family, ${ }^{13}$ which stimulate bone formation. Human recombinant BMP7 (available commercially under the brand name OP1) is used to facilitate fusion of vertebrae to prevent neurological trauma. ${ }^{14}$ rhBMP2 is however more widely used to treat non-union of fractures as it appears to be superior in inducing new bone formation than the other BMPs. ${ }^{15}$ BMP7 has a potential future role in the management of chronic kidney disease through its inhibition of fibrosis and restoration of healthy epithelial cell populations. ${ }^{16-18}$ Mineralisation of the newly formed bone is mediated by bone sialoprotein, ${ }^{14}$ carboxylated osteocalcin $^{19}$ or other cytokines (see Part $I^{\star}$ ). Mapping of these pathways exposed specific receptor binding sites on bone cells which can potentially be activated or blocked in order to either limit bone resorption or accelerate bone formation and mineralisation, impacting directly on the process of bone healing. Carrier systems which deliver bioactive molecules locally, such as the biocoating of implant surfaces with bone morphogenic protein (BMP) (a protein of the TGF $\beta$ family) and other osteogenic cytokines can facilitate integrative bone repair. ${ }^{20}$ Ceramic microsphere carriers are injectable, biodegradable and can be coated to become osteoinductive ${ }^{21,22}$ thereby decreasing the post-infection healing time of bone.

\section{HIV and antiretroviral therapy}

Antiretroviral therapy has changed the fate of HIV infection from a fatal to a manageable chronic disease. With this advancement the co-morbidities resulting from skeletal catabolism are now more prominent in this cohort of patients than in the past. Highly active antiretroviral therapy (HAART), chronic inflammation, the virus itself and dietary factors contribute to bone $\operatorname{loss}^{23}$ and the increase in the incidence of fractures reported in AIDS patients. ${ }^{24}$ A contributing factor is hypovitaminosis D which is prevalent among HIV-positive subjects. ${ }^{25}$ Although the mechanisms involved in the skeletal anabolism of HIV patients on HAART are not fully understood, data now indicate that these patients should be 
included in screening programmes as high risk for osteoporosis. Recent focus on the prominent role the immune system plays in skeletal health makes the influence of the residual immune dysregulation syndrome in treated HIV patients ${ }^{26}$ an unexploited field for research.

\section{Generalised bone forming diseases}

Osteopetrosis is a heterogenous disease with several molecular and genetic defects leading to dysfunctional osteoclasts and unopposed bone formation. Among others, mutations of M-CSF and RANK are involved, as well as over-expression of OPG, as the RANKL/OPG ratio is a major determinant of bone mass. ${ }^{27}$ The clinical severity varies from neonatal onset with bone marrow displacement and fatal pancytopaenia to an incidental finding of bone sclerosis on a radiograph. Repopulation of the bone marrow with normal stem cell populations provides some hope for patients suffering the infant-onset types. The duplication of the signalling peptide (TNFRSF11A) of the gene that encodes for RANK, is associated with a rare panostotic expansile bone disease (distinguished from fibrous dysplasia by an absence of GNAS mutation). ${ }^{28}$ Similar RANK insertion mutations were reported in other expansile bone conditions. ${ }^{29}$ Sclerosing bone dysplasias (sclerosteosis, Worth syndrome and Van Buchem disease) are linked to a genetic mutation which either incapacitates osteocytes to produce sclerostin $^{30}$ or modulates LRP5 or its receptors. ${ }^{31,32}$ Simulation of these mutations through gene transferral may form a basis for the development of therapeutic agents that facilitate bone formation and improve bone healing after surgical procedures.

\section{Paget's disease of bone}

Although a decline in the incidence has been reported in several communities in which Paget's disease is endemic, it remains an important diagnosis in orthopaedic practice. In the advanced stage, it is earmarked by disordered bone formation which leads to skeletal deformity, pathological fractures and neurologic pains and deafness due to compression of nerves which pass through the enlarging bony structures. Paget's disease is the result of a combination of a genetic mutation in the SQSTM1/p62 gene and the impact of an environmental factor, most likely chronic measles virus infection. ${ }^{33}$ The mutation increases the response of osteoclasts to RANK-NF- $\kappa \mathrm{B}$ signalling, leading to osteoclast activation. ${ }^{34}$ This explains the initial resorptive phase of the disease and provides a feasible rationale for the use of bisphosphonates ${ }^{35}$ and denosumab, a RANKL antibody $^{36}$ in the treatment thereof. The abnormal osteoclasts show increased sensitivity to vit $\mathrm{D}_{3}$ and its precursors and other transcription factors. ${ }^{37}$ Elevation of fibroblast growth factor-2 (FGF-2) as well as its influence on osteoblast precursors is related to the disorganised bone formation in the later stages of the disease. ${ }^{38}$

\section{Malignant disease}

Manipulation of the bone microenvironment is a field in which the next thrust in anticancer therapy is predicted. The influence of metastatic deposits of solid malignancies on bone is resorption, bone formation or both. Breast cancer is the prototype of the bone-resorbing and prostate cancer of the bone-forming phenotype. Factors produced by malignant deposits that stimulate osteoclasts include parathyroid hormone related protein (PTHrP), several of the interleukins and RANKL. ${ }^{39}$ The bisphosphonate group of drugs (which block the effects of PTHrP) and denosumab are effective in addressing the skeletal morbidity and hypercalcaemia resulting from the production of osteoclastogenic cytokines by metastatic malignant clones in bone. Inactivation of osteoclasts is however not without complications as patients may develop osteonecrosis (particularly of the jaws), especially those on intravenous nitrogen containing bisphosphonates. ${ }^{40}$ Transforming growth factor $\beta$ (TGF $\beta$ ) is released from the matrix of bone during resorption ${ }^{41}$ and stimulates the elaboration of several catabolic cytokines by tumour cells. The blocking of TGF $\beta$ production in breast cancer through the therapeutic administration of SD-208, an inhibitor of TGF $\beta$, may therefore decrease the skeletal morbidity of breast cancer patients. Prostate cancer metastasis dysregulates bone remodelling and the nett outcome is bone formation, often described as an 'osteoblastic' response. The neoplastic cells produce growth factors such as insulin-like growth factor, plateletderived growth factor, adrenomedullin and a recently discovered vasoactive peptide ET- $1 .{ }^{39}$ The mechanism of stimulation of osteoblasts by ET-1 is unclear. Blocking of the endothelin $\mathrm{A}$ receptor $\left(\mathrm{ET}_{\mathrm{A}} \mathrm{R}\right)$ by atrasentan, an $\mathrm{ET}_{\mathrm{A}} \mathrm{R}$ antagonist, reduced skeletal morbidity in patients with advanced prostate cancer. ${ }^{42}$ This discovery in a rapidly advancing field of research is likely to introduce a new chapter in the management of this disease.

\section{Conclusion}

The study of bone has moved beyond morphology, and exciting fields of research have been uncovered in the last decade. Cell signalling pathways can now be linked to specific disease states and, through intervention, a specific bone phenotype can be induced by cytokine modulation. More studies are, however, required as the long-term effects of interfering in skeletal metabolism, which is part of systemic metabolic pathways, are as yet unknown.

\section{Conflict of interest statement}

The authors have no conflict of interest to declare and received no direct funding for the writing of the article.

\section{References}

1. Evans CH. Gene delivery to bone. Adv Drug Deliv Rev 2012;64:1331-40.

2. Drosse I, Volkmer E, Capanna $\mathrm{R}$ et al. Tissue engineering of bone deficit healing: An update on a multi-component approach Int J Care Injured 2008;39S2:S9-S20. 
3. Eisman JA, Bogoch R, Dell R et al. Making the first fracture the last fracture: ASBMR task force report on secondary fracture prevention. J Bone Min Res 2012;27:2039-46.

4. Burge R, Dawson-Hughes B, Solomon DH et al. Incidence and economic burden of osteoporosis-related fractures in the United States, 2005-2025. J Bone Min Res 2007;22:465-75.

5. McClung MR, Liwiecki EM, Cohen SB et al. Denosumab in post-menopausal women with low bone mineral density. $\mathrm{N}$ Engl J Med 2006;354:821-31.

6. Neer RM, Arnaud CD, Zanchetta JR et al. Effect of parathyroid hormone (1-34) on fractures and bone mineral density in postmenopausal women with osteoporosis. New Engl J Med 2001;344:1434-41.

7. Kim JH, Liu X, Wang J et al. Wnt signalling in bone formation and its therapeutic potential for bone diseases. Ther Adv Musculoslelt Dis 2013;5:13-31

8. Street J, Bao M, deGuzman L et al. Vascular endothelial growth factor stimulates bone repair by promoting angiogenesis and bone turnover. Proc Natl Acad Sci USA 2002;99:9656-61.

9. Yadav VK, Balaji S, Suresh PS et al. Pharmacological inhibition of gut-derived serotonin synthesis is a potential bone anabolic treatment for osteoporosis. Nat Med 2010;16:308-12.

10. Abu-Amer Y. NF-kB signalling in bone resorption. Osteoporos Int 2013; doi:10.1007/ s00198-013-2313-x

11. Rossini M, Gatti D, Adami S. Involvement of Wnt $/ \beta$-catenin signalling in the treatment of osteoporosis. Calcif Tissue Int 2013;93:121-32.

12. Weitzmann MN. Review Article. The role of inflammatory cytokines, the RANKL/OPG axis, and the immunoskeletal interface in physiological bone turnover and osteoporosis. Scientifica 2013; doi.org/10.1155/2013/125705

13. Chen D, Zhoa M, Mundy GR. Bone morphogenetic proteins. Growth Factors 2004;22:233-41.

14.. Vaccaro AP, Whang PG, Patel T et al. The safety and efficacy of OP-1 (rhBMP-7) as a replacement for iliac crest autograph for posterolateral lumbar arthrodesis: minimum 4-year follow-up of a pilot study. Spine J 2008;8:457-65.

15. Even J, Eskander M, Kang J. Bone morphogenetic protein in spine surgery: current and future uses. J Am Acad Orthop Surg 2012;20:547-52

16. Gould SE, Day M, Jones SS et al. BMP-7 regulates chemokine, cytokine, and hemodynamic gene expression in proximal tubule cells. Kidney Int 2002;61:51-61.

17. Kalluri R, Weinberg RA. The basics of epithelial-mesenchymal transition. J Clin Invest 2009;119:1420-28.

18. Zeisberg M, Bottiglio C, Kumar $\mathrm{N}$ et al. Bone morphogenic protein-7 inhibits progression of chronic renal fibrosis associated with two genetic mouse models. Am J Physiol Renal Physiol 2003;285:F1060-F1067.

19.. Gordon JA, Tye CE, Sampaio AV et al. Bone sialoprotein expression enhances osteoblast differentiation and matrix mineralisation in vitro. Bone 2007;41:462-73.

20. Karsenty G, Ferron M. The contribution of bone to whole organ physiology. Nature 2012;481:314-20.

21. Jennisen HP. Accelerated and improved osseointegration of implants biocoated with bone morphogenetic protein 2 (BMP2). Ann NY Acad Sci 2002;961:139-42.

22. Tiffany NV, Kasper FK, Mikos AG. Strategies for controlled delivery of growth factors and cells for bone regeneration. Adv Drug Deliv Rev 2012;64:1292-1309.

23. de Mendez Barbosa EG, de Paulo FJ, Machado AA et al. Impact of antiretroviral therapy on bone metabolism markers in HIVseropositive patients. Bone 2013;57:62-67.
24. Shiau S, Broun EC, Arpadi SM, Yin MT. Incident fractures in HIV-infected individuals: a systematic review and metaanalysis. AIDS 2013;27:1949-57.

25. Pinzone MR, Di Rosa M, Malaguarnera M et al. Vit D deficiency in HIV infection: an underestimated and undertreated epidemic. Eu Rev Med Pharmacol Sci 2013;17:1218-32.

26. Lederman MM, Funderburg NT, Sekaly RP et al. Residual immune dysregulation syndrome in treated HIV infection. Adv Immunol 2013;119:51-83.

27. Hofbauer LC, Shoppet M. Clinical implications of the osteoprotegerin/RANKL/RANK system for bone and vascular diseases. JAMA 2004;292:490-45.

28. Schafer AL, Mumm S, El-Sayed I et al. Panostotic expansile bone disease with massive jaw tumor formation and a novel mutation in the signalling peptide of RANK. J Bone Miner Res 2014;29:911-21.

29. Crockett JC, Mellis DJ, Scott DI et al. New knowledge on critical osteoclast formation and activation pathways from study of rare genetic diseases of osteoclasts: focus on the RANK/RANKL axis. Osteoporos Int 2011;22:1-20.

30. Moester MJ, Papapoulos SE, Lòwik CW et al. Sclerostin: current knowledge and future perspectives. Calcif Tissue Int 2010;87:99107.

31. Wang $\mathrm{C}$, Zhang BH, Zhang $\mathrm{H}$ et al. The A242T mutation in the low-density lipoprotein receptor-related protein 5 gene in one Chinese family with osteosclerosis. Intern Med 2013;52:187-92.

32. van Bezooijen RL, Bronckers AL. Gortzak RA et al. Sclerostin in mineralized matrices and van Buchems disease. J Dent Res 2009;88:569-74.

33. Galston DL, Roodman GD. Pathobiology of Paget's disease of bone. J Bone Metab 2014;21:85-98.

34. Ralston SH. Pathogenesis of Paget's disease of bone. Bone 2008;43:819-825.

35. Kim KC. Intravenous Zoledronate for a patient with Paget's disease. J Bone Metab 2014;21:223-26.

36. Grasemann C, Schündeln MM, Hövel M et al. Effects of RANKligand antibody (denosumab) treatment on bone turnover markers in a girl with juvenile Paget's disease. J Clin Endocrinol Metab 2013;98:3121-26.

37. Sun $\mathrm{Q}$, Sammut $\mathrm{B}$, Wang FM et al. TBK1 mediates critical effects of measles virus nucleocapsid protein (MVNP) on pagetic osteoclast formation. J Bone Miner Res 2014;29:90-102.

38. Sundaram K, Senn J, Reddy SV. SOCS-1/3 participation in FGF2 signalling to modulate RANK ligand expression in Paget's disease of bone. J Cell Biochem 2013;114:2032-38.

39. Guise TA, Mohammed KS, Clines G et al.Basic mechanisms responsible for osteolytic and osteoblastic metastases. Clin Cancer Res 2006 doi:10.1158/1078-0432.CCR-06-1007

40. Woo SB, Hellstein JW, Kalmar JR. Systematic review: Bisphosphonates and osteonecrosis of the jaws. Ann Intern Med 2006;144:753-61.

41. Dallas SL, Rosser JL, Mundy GR, Bonewald LF. Proteolysis of latent growth factor- $\beta$ (TGF- $\beta$ )- binding protein-1 by osteoclasts: a cellular mechanism for the release of TGF- $\beta$ from bone matrix. J Biol Chem 2002;277:21352-60.

42. Carducci MA, Padley RJ, Breul J et al. Effect of endothelin-A receptor blockage with atrasentan on tumor progression in men with hormone refractory prostate cancer: a randomized, phase II placebo-controlled trial. J Clin Oncol 2003;21:679-89.

This article is also available online on the SAOA website (www.saoa.org.za) and the SciELO website (www.scielo.org.za). Follow the directions on the Contents page of this journal to access it. 